\section{ORIGINAL RESEARCH}

J. Klisch

A. Turk

R. Turner

H.H. Woo

D. Fiorella

\title{
Very Late Thrombosis of Flow-Diverting Constructs after the Treatment of Large Fusiform Posterior Circulation Aneurysms
}

BACKGROUND AND PURPOSE: The PED is a new endoluminal construct designed to exclude aneurysms from the parent cerebrovasculature. We report the very late ( $>1$ year) thrombosis of PED constructs in 2 patients.

RESULTS: Two patients with very large fusiform basilar trunk aneurysms underwent parent artery reconstruction with the PED. Both patients were maintained on dual antiplatelet therapy throughout the first year following treatment. Follow-up conventional angiography, performed 12 months after treatment, demonstrated, in both patients, thrombosis of most of the aneurysm with minimal residual flow through the construct and into the aneurysm fundus. In response to the residual filling, clopidogrel was discontinued (aspirin therapy was maintained). Both patients presented with symptomatic acute occlusions of the PED constructs within 14 days of clopidogrel discontinuation.

DISCUSSION: Patient 1 presented with constitutional symptoms that progressed to severe headache without other neurologic signs or symptoms. Occlusion of the PED construct was confirmed with conventional angiography. MR imaging demonstrated no evidence of infarction or parenchymal injury. The headaches were managed successfully with steroid therapy. Patient 2 presented with a syndrome of acute basilar occlusion with brain stem stroke. Complete occlusion was confirmed on angiography. Emergent thrombolysis with mechanical revascularization was performed successfully; however, the patient ultimately succumbed to the infarction.

CoNCLUSIONS: It appears that flow-diverting constructs built across large circumferential aneurysms may remain thrombogenic for much longer than conventional intracranial or peripheral bare metal stents. Constructs in these patients may remain susceptible to very late thrombosis, $>1$ year after implantation. These patients likely require long-term dual antiplatelet therapy ( $>1$ year) to provide adequate prophylaxis against thrombosis. If these types of aneurysms demonstrate persistent residual filling months after PED reconstruction, operators should consider the placement of additional devices as an alternative to the discontinuation of 1 of the antiplatelet medications.

ABBREVIATIONS: AICA = anterior inferior cerebellar artery; CTA = CT angiography; $\mathrm{PA}=$ posterioranterior; PED = Pipeline embolization device

$\mathbf{F}$ ow diverters are flexible microcatheter-delivered selfexpanding braided stents that provide 30\%-35\% metal surface-area coverage when deployed in appropriately sizematched vessels. These high-metal-surface-area constructs are designed to achieve exclusion of cerebral aneurysms through a combination of flow diversion and parent artery reconstruction. These devices have recently received full CE Mark approval for commercialization within the European Union, and they remain investigational within the United States. The reported periprocedural and midterm follow-up results to date have been extremely impressive, ${ }^{1-4}$ marked by very high rates of curative parent artery reconstruction achieved

Received December 2, 2010; accepted after revision January 29, 2011.

From the Department of Neuroradiology (J.K.), HELIOS Klinikum, Erfurt, Germany; Department of Neurosurgery and Neuroradiology (A.T., R.T.), Cerebrovascular Center, Medical University of South Carolina, Charleston, South Carolina; and Department of Neurosurgery (H.H.W., D.F.), Stony Brook University Medical Center, Cerebrovascular Center, Stony Brook, New York.

Please address correspondence to David Fiorella, MD, PhD, Department of Neurosurgery, Cerebrovascular Center, State University of New York at Stony Brook, Stony Brook University Hospital, Health Sciences Center 080, Stony Brook, NY; e-mail: dfiorella@ notes.cc.sunysb.edu

Indicates open access to non-subscribers at www.ajnr.org

DOI 10.3174/ajnr.A2571 with a reasonable level of treatment-associated morbidity and mortality. However, as a very new technology, long-term follow-up results are lacking.

Very late thrombosis is defined in the coronary literature as stent occlusion occurring $>1$ year after implantation. Very late thrombosis of bare metal coronary stents represents an extremely uncommon complication. ${ }^{5}$ We recently reported the very late thrombosis of a PED construct (eV3/Covidian, Irvine, California) applied to treat a long-segment circumferential aneurysm of the vertebral artery, occurring almost 2 years after implantation. ${ }^{6}$

We report 2 additional cases of very late thrombosis of $\mathrm{PED}$ constructs placed to treat large circumferential posterior circulation aneurysms. We review the implications of these observations for the management of patients with these very complex and challenging aneurysms.

\section{Case Reports}

\section{Patient 1}

Presentation. An adult female patient with a family history of aneurysmal subarachnoid hemorrhage presented with a history of progressively worsening intractable headache. An initial evaluation for meningitis was negative, and ultimately cross-sectional brain imaging was performed. CT, CTA, and MR imaging demonstrated a 
partially thrombosed very large fusiform aneurysm circumferentially involving a 22-mm segment of the basilar trunk (Fig 1).

Treatment. No conventional open surgical or endovascular options for treatment were evident. For this reason, we provided the patient with the option of being treated with the PED under an institutional review board approval and US Food and Drug Administration-approved compassionate use provision, allowing for the 1 time use of an unapproved device. She provided full informed consent to proceed.

After pretreatment with aspirin and clopidogrel, the aneurysm was reconstructed with a series of 4 sequentially placed Pipeline devices $(3.25 \times 20 \mathrm{~mm}, 3.5 \times 20 \mathrm{~mm}, 3.5 \times 16 \mathrm{~mm}$, and $3.5 \times 16 \mathrm{~mm})$, which composed a construct spanning from the distal to the proximal basilar trunk. The treatment was accomplished without complications, and the patient emerged from general anesthesia neurologically intact. She was maintained on dual antiplatelet therapy for 1 year. Her presenting headaches resolved after the treatment, and she remained asymptomatic and neurologically healthy. Follow-up angiography was performed 3 and 12 months after the initial treatment. The initial follow-up angiogram demonstrated a small amount of residual filling of the aneurysm through the midportion of the construct, which decreased slightly at 12 months. Cone-beam CTA (DynaCT; Siemens, Erlangen, Germany) performed at the time of the second angiography demonstrated that the aneurysm-intraaneurysmal thrombus mass had not significantly decreased in volume since the original treatment. Moreover, contrast material was seen penetrating into the intra-aneurysmal thrombus mass.

On the basis of this finding, we recommended that the patient discontinue clopidogrel, with the intention that the small amount of residual filling would resolve with a single antiplatelet agent therapy (aspirin).

Very Late Thrombosis. Approximately 5 days after discontinuing clopidogrel, the patient began to experience constitutional flulike symptoms, which were accompanied by a progressive headache. She described the headache as similar to those she had experienced before PED reconstruction. She denied any additional focal neurologic symptoms.

Follow-up angiography was performed emergently, demonstrating complete occlusion of the aneurysm and basilar artery trunk over the entire reconstructed segment. The vertebral arteries provided flow to the proximal vertebrobasilar system. The distal basilar artery was perfused by a large right posterior communicating artery. MR imaging demonstrated a large residual aneurysm mass with thrombus distributed throughout the fundus. The collective aneurysmthrombus mass measured $17 \mathrm{~mm}$ in greatest anteroposterior diameter. Diffusion and long TR-weighted sequences showed no evidence of acute or chronic parenchymal infarction.

Patient Management. The patient was maintained on aspirin therapy. Her headaches were successfully managed with analgesics and an aggressive course of corticosteroids. She remains neurologically intact.

\section{Patient 2}

Presentation. An adult male patient presented with progressive headache, double vision, and hyperreflexia. Cross-sectional imaging demonstrated a very large fusiform aneurysm circumferentially involving a 25-mm segment of the proximal basilar trunk with incorporation of the vertebrobasilar junction (Fig 2).

Treatment. No conventional open surgical or endovascular options for treatment were evident. An initial attempt at endovascular reconstruction with 2 Solitaire stents (ev3, Irvine, California) was unsuccessful. For this reason, the patient was provided with the option to be treated with flow diversion. The treatment was performed in Europe after the PED was commercially available under CE Mark approval. He provided full informed consent to proceed.

After pretreatment with aspirin and clopidogrel, the aneurysm was reconstructed with a series of 9 sequentially placed Pipeline devices that composed a construct spanning from the distal basilar trunk into the distal right vertebral artery. The distal left vertebral artery was occluded with bare platinum embolization coils. The treatment was accomplished without complications, and the patient emerged from general anesthesia neurologically at baseline. He was maintained on dual antiplatelet therapy for 11 months.

Follow-up angiography performed 11 months after the initial treatment demonstrated residual filling of the aneurysm through the left lateral aspect of the construct with irregular penetration of contrast into the thrombus mass.

On the basis of this finding, the clopidogrel was discontinued with the expectation that this would allow the persistent residual filling of the aneurysm to proceed to thrombosis. The patient was maintained on aspirin therapy.

Very Late Thrombosis. Two weeks after the discontinuation of clopidogrel therapy (and 12 months after the initial treatment), the patient re-presented with a basilar occlusion syndrome consisting of tetraparesis progressing to coma. Conventional angiography demonstrated complete occlusion of the distal right vertebral artery at the level of the construct. The right posterior inferior cerebellar artery provided some collateral flow to the cerebellar hemispheres via leptomeningeal collaterals to the AICA. The entire reconstructed segment of the basilar artery was completely occluded.

Patient Management. The patient was brought emergently to the endovascular suite for revascularization of the occluded construct. This was achieved with intravenous eptifibitide, intra-arterial tissue plasminogen activator, and adjunctive mechanical thrombectomy using the Solitaire device (eV3). Despite successful re-vascularization, the patient had a large posterior circulation infarction that required suboccipital decompression. He remained comatose and ultimately died.

\section{Discussion}

The most important points derived from the reported cases are the following: 1) PED constructs built across large fusiform aneurysms that circumferentially involve long arterial segments $(>20 \mathrm{~mm})$ may remain thrombogenic long after implantation. 2) Correspondingly, these patients may require long-term $(>1$ year) dual antiplatelet therapy after treatment to provide adequate prophylaxis against construct thrombosis. This regimen of antiplatelet therapy is far longer than that traditionally required for the prophylaxis of other conventional self-expanding intracranial stents. ${ }^{3}$ If patients with such constructs demonstrate persistent residual aneurysm filling after treatment, the placement of additional devices over the entry remnant may represent an alternative to clopidogrel discontinuation as a strategy for achieving complete (curative) exclusion of the aneurysm.

\section{Etiology of Very Late Thrombosis of a PED Construct}

The initial experience with the PED has been extremely promising, characterized by very high levels of curative parent artery reconstruction achieved with relatively low levels of 

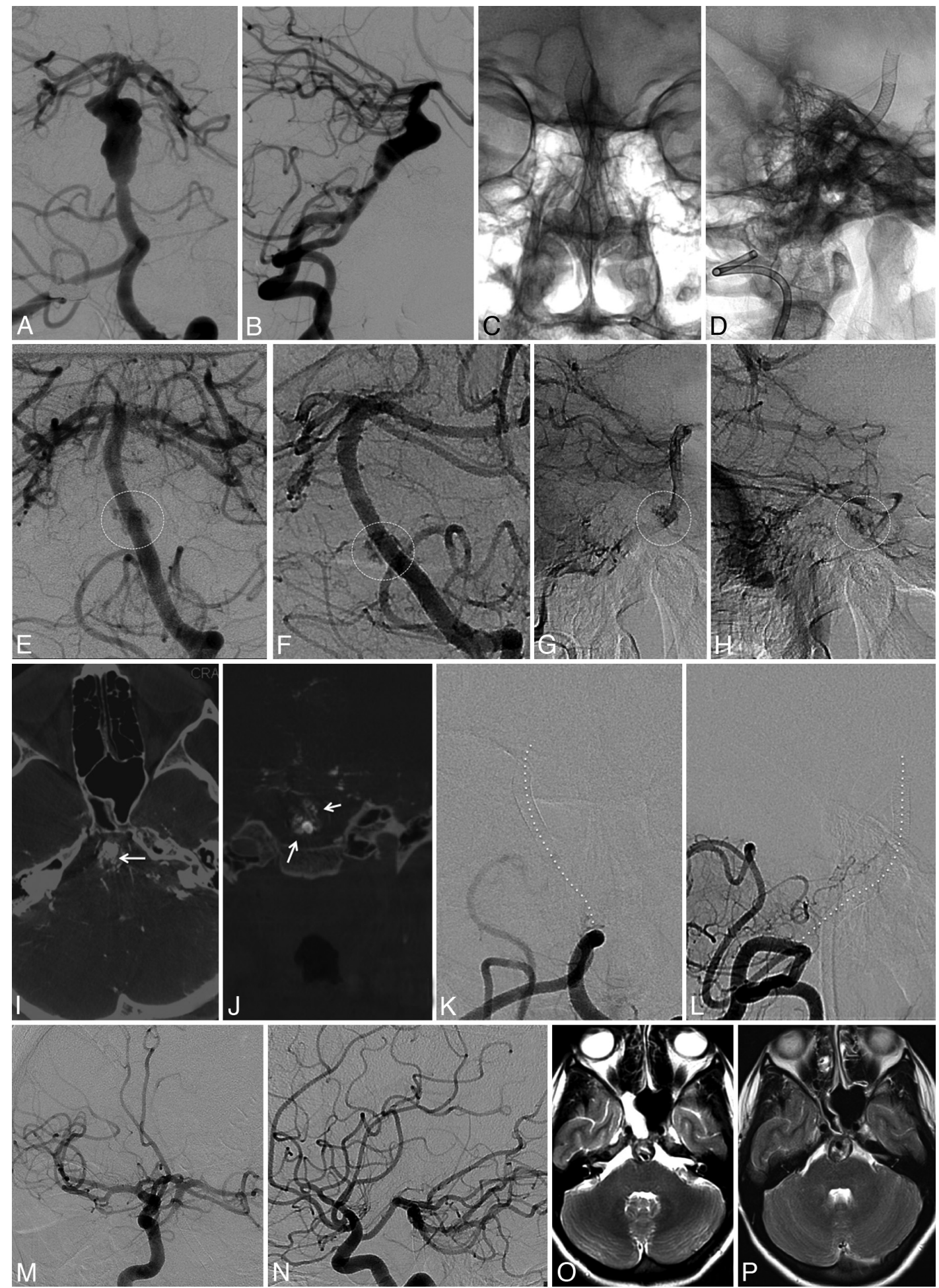

Fig 1. Subtracted PA $(A)$ and lateral $(B)$ pretreatment angiographic projections demonstrate a fusiform aneurysm with incorporation of a 20-mm segment of the midbasilar trunk. The trajectory of the distal basilar artery appears displaced posteriorly and to the right with respect to the trajectory of the proximal basilar trunk. Native $P A(C)$ and lateral $(D)$ projections following treatment demonstrate a 4-PED construct bridging the aneurysmal segment of the basilar trunk. Subtracted PA $(E)$ and slightly oblique $(F)$ projections from 3- $(E)$ and 12-month $(F)$ follow-up angiograms demonstrate an irregularly marginated neck entry remnant along the posterior aspect of the midconstruct, demarcated by dotted circles. Subtracted capillary $(G)$ and late venous $(H)$ phase lateral images from the 12-month follow-up study demonstrate the margins of the residual pocket, demarcated by dotted circles, to be somewhat irregular, with an appearance suggesting gradual penetration into the thrombosed portion of the aneurysm during the later phases of the angiogram. Axial (/) and coronal $(\mathcal{J}$ reformations of source data derived from a cone-beam CTA (DynaCT) following an intra-arterial contrast injection (arrows) demonstrate transit of contrast material through the PED construct with irregular penetration into the intra-aneurysmal thrombus mass. In addition, the overall size of the aneurysm-intra-aneurysmal thrombus mass has not regressed in comparison with original cross-sectional imaging studies obtained before treatment. Subtracted PA $(K)$ and lateral $(L)$ images from an angiogram obtained 10 days after discontinuation of clopidogrel demonstrate complete occlusion of the PED construct. The normal trajectory of the reconstructed basilar artery is demarcated by a dotted line on each image. Subtracted oblique $(M)$ and lateral $(N)$ images demonstrate a large patent right posterior communicating artery providing perfusion of the distal basilar artery and basilar apex. Long TR-weighted axial imagings performed before $(0)$ and 13 months after $(P)$ treatment confirm that the size of the aneurysm-thrombus mass complex had not regressed to any extent after the initial PED reconstruction, suggesting continued viability of the aneurysm despite PED treatment and near-complete thrombosis on angiography during the next 12 months. 

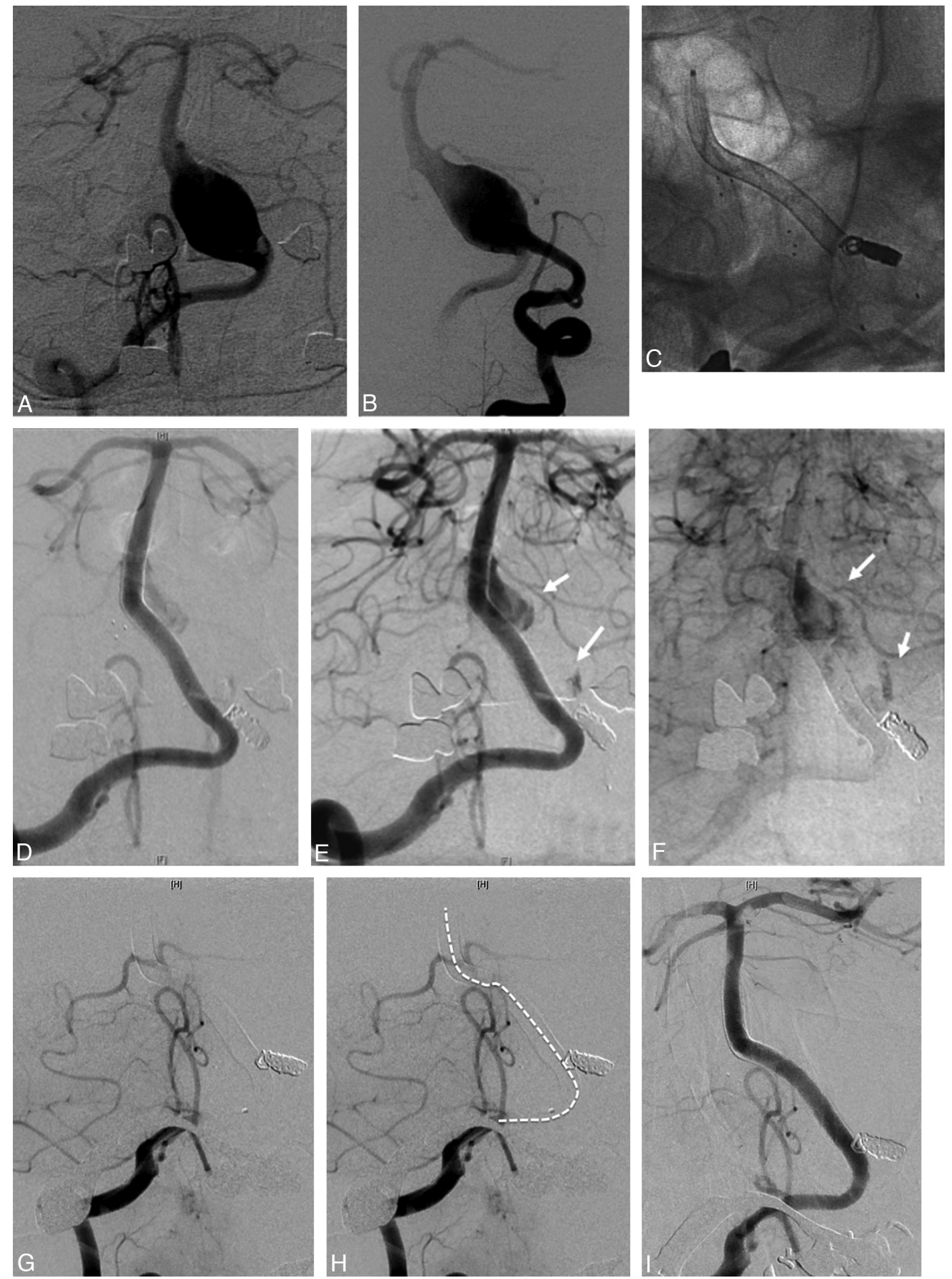

Fig 2. Subtracted $P A(A)$ and oblique $(B)$ images demonstrate a fusiform aneurysm circumferentially incorporating a $25-\mathrm{mm}$ segment of the basilar artery, extending from just beyond the origin of the AICAs proximally to involve the vertebrobasilar junction. C. Native oblique projection following reconstruction of the vertebrobasilar junction with a PED construct extending from the distal right vertebral artery to the distal basilar artery and coil deconstruction of the distal left vertebral artery. Additional markers within the aneurysm fundus demarcate the ends of 2 Solitaire stents, which had been deployed during a prior (unsuccessful) attempt at treatment. Subtracted angiographic images during the early $(D)$ and mid $(E)$ arterial phases as well as the venous $(F)$ phase from an 11-month follow-up angiogram demonstrate residual filling of the aneurysm via a neck-entry remnant adjacent to the left AICA origin. Later phase imaging demonstrates penetration of contrast material (arrows) deep into the intra-aneurysmal thrombus mass. Subtracted PA image (G) from a 12-month follow-up angiogram demonstrates complete occlusion of the PED construct. $H$, The normal trajectory of the reconstructed distal right vertebral artery and basilar trunk is demarcated in the adjacent figure by a dotted white line. Subtracted PA angiographic image obtained during the emergent revascularization of the PED construct demonstrates restoration of the patency of the construct with a small amount of residual thrombus material within the basilar apex and proximal posterior cerebral arteries. 
treatment-associated complications - even when the device is applied for the treatment of the most challenging cerebral aneurysms. ${ }^{1-4}$ However, as a new device, very little long-term clinical or angiographic follow-up data are available. There are also no data to support a standardized approach to the management of antiplatelet therapy following the implantation of these devices-especially when multidevice constructs are built across more extensive and complex aneurysms.

We recently presented a case report of the very late thrombosis of a PED construct that ultimately resulted in a fatal posterior circulation infarct. ${ }^{6}$ Given the very delayed presentation of this patient (23 months), the etiology of the occlusion was somewhat difficult to determine. One of the mechanisms considered, as a potential stimulus for the construct occlusion, was thrombosis following the recent alteration of the patient's antiplatelet therapy. However, given the extensive time interval between the initial treatment and the observed thrombosis, this was thought unlikely.

In the 2 index cases presented, the etiology of very late occlusion is somewhat more straightforward, and these 2 cases potentially provide insight into the prior case. Both of the index patients underwent follow-up conventional angiography at 1 year that demonstrated progressive thrombosis of the aneurysms to near-complete occlusion with minimal residual filling through the constructs. In both cases, the reconstructed parent artery lumen was widely patent without any evidence of in-construct stenosis or thrombus formation. In both cases, clopidogrel was discontinued with the intention of allowing these neck entry remnants to proceed to thrombosis. Both patients then presented acutely with thrombosis of their constructs several days after clopidogrel discontinuation and only weeks after the 1-year control angiogram demonstrated full patency of the parent artery. These findings essentially provide conclusive evidence that these very late occlusions were directly attributable to thrombosis of the constructs after clopidogrel discontinuation.

Given the recent reports of delayed aneurysm rupture, the findings of residual aneurysm filling with irregular penetration of contrast material into the intra-aneurysmal thrombus mass provided an indication that these aneurysms were potentially unstable. ${ }^{7-10}$ As such, the discontinuation of clopidogrel therapy represents an attractive, noninvasive means by which to accelerate the thrombosis of these persistent neck-entry remnants. However, in light of the present findings, operators might consider maintaining dual antiplatelet therapy in these patients and placing additional flow-diverting devices within the pre-existing construct as a means of obliterating the persisting entry remnants.

\section{Hypothetical Reasons for the Retained Thrombogenicity of Long-Segment PED Constructs}

Most data from the coronary literature have demonstrated neointimal overgrowth to be complete within 6 months of the implantation with bare metal stents. ${ }^{11,12}$ Although there has been a significant amount of attention paid to the very late $(>1$ year) thrombosis of drug-eluting stents implanted within the coronary arteries, very late thrombosis of bare metal stents is exceedingly rare, with only 21 cases reported in the medical literature (as of 2009) despite millions of bare metal coronary stents having been implanted worldwide. ${ }^{5}$ Interestingly, a ret- rospective analysis of these rare cases of delayed bare metal coronary stent thrombosis demonstrated no relationship to antiplatelet therapy discontinuation or alteration. ${ }^{5}$

These observations are in direct opposition to the current experience with these much more extensive intracranial flowdiverting constructs. First, the overall number of very large circumferential posterior-circulation aneurysms that have been treated with the PED is comparatively very small. That 3 cases of very late thrombosis have already been reported indicates that the incidence of this occurrence with flow diverters cannot be extrapolated from observations derived from the application of bare metal stents placed within the coronary circulation or conventional coil-assist stents implanted within the cerebrovasculature. Second, in all 3 cases, the construct thrombosis was temporally associated with alteration or discontinuation of an established regimen of antiplatelet therapy. Thus, these observations indicate that in the index cases, construct thrombosis was directly related to antiplatelet medication disruption and was, therefore, indicative of the retained thrombogenicity of these more complex long-segment constructs, which are built across fusiform aneurysms. It is very likely that constructs in circumferential apposition to long segments of intra-aneurysmal thrombus may "endothelialize" at a rate far slower than that observed when a similarly sized construct is placed across a noncircumferential aneurysm. When used to treat more conventional noncircumferential (ie, saccular) sidewall aneurysms, the constructs are primarily in apposition to a surface area of intact parent artery rather than "free" intra-aneurysmal thrombus. A final consideration is that the progressive thrombosis of these entry remnants following clopidogrel discontinuation may occur in an overexuberant manner, which leads to the uncontrolled extension of the evolving thrombus into the lumen of the construct, ultimately leading to occlusion.

\section{Conclusions}

We report 2 additional cases of very late ( $>1$ year) thrombosis of a PED construct. It appears that PED constructs built across very large fusiform aneurysms that circumferentially involve long segments $(>20 \mathrm{~mm})$ of the parent artery likely require much longer to become covered with neoendothelialneointimal tissue than conventional bare metal stents. As such, these long-segment constructs likely retain their thrombogenicity for a much longer time. For these reasons, patients treated with such constructs may require long-term ( $>1$ year), possibly life-long, dual antiplatelet medications for prophylaxis against thrombosis. When these patients show persistent aneurysm filling several months after treatment, operators should consider the placement of an additional device over the region of the neck entry remnant as an alternative to the discontinuation of clopidogrel to achieve a curative reconstruction of the aneurysm.

Disclosures: Joachim Klisch, Consultant: ev3. Details: Proctoring in Europe for Pipeline and Onyx. Aquilla Turk, Research Support (including provision of equipment or materials): Codman/Micrus, Penumbra, NFocus Medical, Pulsar Vascular, Mindframe. Details: Codman/Micrus-\$10,000 $\$ 25,000$ for fellow and physician training courses in animal laboratory; Penumbra- $\$ 10,000$ to evaluate aneurysm coil in animal model; NFocus- $>\$ 25,000$ to evaluate aneurysm device in animal model; Pulsar Vascular- $>\$ 25,000$ to evaluate aneurysm assist device in animal model; Mindframe- $>\$ 25,000$ to evaluate stroke device. Consultant: Boston Scientific, Codman/Micrus, Penumbra, NFocus Medical, Pulsar Vascu- 
lar, Mindframe. Details: $<\$ 10,000$ for all companies. Other Financial Interests: Boston Scientific. Details: $>\$ 10,000$ to proctor physicians during first stent case. Raymond Turner, Research Support (including provision of equipment or materials): MindFrame Inc, Salient Surgical. Micrus. Details: Research Grants, provide devices for Research Projects. Speaker Bureau: Micrus/Codman, MicroVention, Penumbra. Details: On a per-project basis. Consultant: Micrus/Codman, MicroVention, Salient Surgical, Mindframe. Details: Consultant on a per-project basis. Henry Woo, Research Support (including provision of equipment or materials): Siemens. Details: Research grants for imaging research. Consultant: Micrus Endovascular. Details: General consulting and intellectual property related to a device designed to treat acute ischemic stroke. David Fiorella, Research Support (including provision of equipment or materials): National Institutes of Health, Siemens Medical. Speaker Bureau: Micrus-Codman-Johnson \& Johnson, MicroVention. Details: Significant conflict $(>\$ 10,000)$ for both. Consultant: Micrus-Codman, MicroVention, eV3, Nfocus. Details: Micrus-Codman; MicroVention, significant conflict $(>\$ 10,000)$; ev3, unpaid consultant and proctor; NFocus, unpaid consultant. Ownership Interest: Micrus-Codman. Details: Royalties related to ReVasc Inc, significant conflict $(>\$ 10,000)$.

\section{References}

1. Szikora I, Berentei Z, Kulcsar Z, et al. Treatment of intracranial aneurysms by functional reconstruction of the parent artery: the Budapest experience with the Pipeline embolization device. AJNR Am J Neuroradiol 2010; 31:1139-47

2. Fiorella D, Woo HH, Albuquerque FC, et al. Definitive reconstruction of circumferential, fusiform intracranial aneurysms with the Pipeline embolization device. Neurosurgery 2008;62:1115-20, discussion 1120-21

3. Fiorella D, Kelly ME, Albuquerque FC, et al. Curative reconstruction of a giant midbasilar trunk aneurysm with the Pipeline embolization device. Neurosurgery 2009;64:212-17, discussion 217

4. Lylyk P, Miranda C, Ceratto R, et al. Curative endovascular reconstruction of cerebral aneurysms with the Pipeline embolization device: the Buenos Aires experience. Neurosurgery 2009;64:632-42, discussion 642-43, quiz N636

5. Lemesle G, Pinto Slottow TL, Waksman R. Very late stent thrombosis after bare-metal stent implantation: case reports and review of the literature. J Invasive Cardiol 2009;21:E27-32

6. Fiorella D, Hsu D, Woo HH, et al. Very late thrombosis of a Pipeline embolization device construct: case report. Neurosurgery 2010;67(3 suppl operative) E313-14, discussion E314

7. Turowski B, Macht S, Kulcsar Z, et al. Early fatal hemorrhage after endovascular cerebral aneurysm treatment with a flow diverter (SILK-Stent): do we need to rethink our concepts? Neuroradiology 2011;53:37-41. Epub 2010 Mar 26

8. Kulcsar Z. Rupture of previously unruptured aneurysms after flow diversion treatment. In: Proceedings of the ABC/WIN Conference, Val d'Isere, France. February 23-26, 2010

9. Kulcsar Z, Houdart E, Bonafe A, et al. Intra-aneurysmal thrombosis as a possible cause of delayed aneurysm rupture after flow-diversion treatment. $A J N R$ Am J Neuroradiol 2011;32:20-25. Epub 2010 Nov 11

10. O'Kelly CJ, Krings T, Fiorella D, et al. A novel grading scale for the angiographic assessment of intracranial aneurysms treated using flow diverting stents. Interv Neuroradiol 2010;16:133-37. Epub 2010 Jul 19

11. Farb A, Sangiorgi G, Carter AJ, et al. Pathology of acute and chronic coronary stenting in humans. Circulation 1999;99:44-52

12. Grewe $\mathrm{PH}$, Deneke T, Machraoui A, et al. Acute and chronic tissue response to coronary stent implantation: pathologic findings in human specimen. $\mathrm{J} \mathrm{Am}$ Coll Cardiol 2000;35:157-63 